CRYSTALLOGRAPHIC COMMUNICATIONS

ISSN 2056-9890

Received 20 July 2021

Accepted 28 July 2021

Edited by J. Ellena, Universidade de Sâo Paulo, Brazil

Keywords: crystal structure; carbazole; Cadogan reaction.

CCDC reference: 2099768

Supporting information: this article has supporting information at journals.iucr.org/e

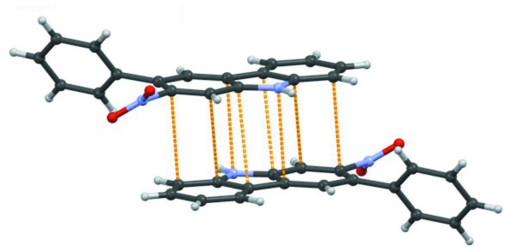

OPEN $\odot$ ACCESS

\section{Crystal structure, Hirshfeld surface and photo- physical analysis of 2-nitro-3-phenyl-9H-carbazole}

\author{
Neetu Singh, Ga Hee Noh, Hyoung-Ryun Park* and Junseong Lee* \\ Department of Chemistry, Chonnam National University, Gwangju, 61186, Republic of Korea. *Correspondence e-mail: \\ hrpark@chonnam.ac.kr, leespy@chonnam.ac.kr
}

The title compound, $\mathrm{C}_{18} \mathrm{H}_{12} \mathrm{~N}_{2} \mathrm{O}_{2}$, was synthesized from a dinitrobiphenylbenzene derivative using a novel modification of the Cadogan reaction. The reaction has several possible ring-closed products and the title compound was separated as the major product. The X-ray crystallographic study revealed that the carbazole compound crystallizes in the monoclinic $P \overline{1}$ space group and possesses a single closed Cadogan ring. There are two independent molecules in the asymmetric unit. In the crystal, the molecules are linked by $\mathrm{N}-\mathrm{H} \cdots \mathrm{O}$ hydrogen bonding.

\section{Chemical context}

Carbazole consists of two benzene ring fused on either side of a central pyrrole ring and is also known as dibenzopyrrole or diphenylenimine. This N-containing heterocyclic compound was discovered by Graebe and Glaser in 1872 (Collin et al., 2006). Carbazoles represent an important class of heterocycles with several advantages. By the introduction of substituents in the carbazole fragment at the nitrogen atom and the aromatic framework at positions 3 and 6 , the photophysical properties can be modified (Srivastava \& Chakrabarti, 2017; Sun et al., 2015). The high stability and redox potential property of carbazole-based polymers compared with other conducting polymers has attracted a great attention (Nandy et al., 2014; Bashir et al., 2015; Sutanto et al., 2021; Niu et al., 2021). Carbazole-based ligands exhibit high hole-transporting mobility and strong absorption in the UV-visible spectroscopic region, and therefore show good electro- and photoactive properties (Yavuz et al., 2001). Polycyclic compounds containing two pyrrole rings have become widely used because of their good charge-transfer properties and the feasibility of tuning the electronic levels in the compound for different types of applications (Wakim et al., 2008; Reig et al., 2015; Xiang et al., 2018; Zhang et al., 2018; Szafraniec-Gorol et al., 2021), These types of compounds are therefore excellent candidates for applications such as OLEDs (organic lightemitting diodes; Svetlichnyi et al., 2010; Oda et al., 2021; Zhou et al., 2021; Bao et al., 2020), DSSCs (dye-sensitized solar cells; Zhang et al., 2009; Li et al., 2018; Lokhande et al., 2019), OPV (organic photovoltaics; Chan et al., 2013; Yang et al., 2020) and OFETs (organic field-effect transistors; Reig et al., 2015; Chen et al., 2020; Koli et al., 2020).

The title compound was isolated as an intermediate in the middle of the synthetic route for the synthesis of double Cadogan-fused carbazoles. The reaction between 1,3-dinitrodiphenylbenzene and triphenylphosphine using the solvent 
$o$-dichlorobenzene resulted in a mixture of single- and doubleCadogen ring-closure products. First, a dinitro compound was obtained by a nitration reaction and in the second step, performing double Suzuki coupling reaction on 1,5-dibromo2,4-dinitrobenzene and benzeneboronic acid gave a terphenyl compound. Then, in the final step, a single Cadogan ring closure was performed to obtain the title compound, $\mathbf{1}$.<smiles>O=[N+]([O-])c1cc2[nH]c3ccccc3c2cc1-c1ccccc1</smiles>

\section{Structural commentary}

Structural analysis confirmed the formation of a single Cadogan ring major product, i.e. carbazole with a nitro group at the 2-position, and a phenyl group at the 3-position. The molecular structure of compound $\mathbf{1}$ is shown in Fig. 1. There are two independent molecules in the asymmetric unit in which the dihedral angles between the carbazole ring system (r.m.s. deviations of 0.001 and $0.002 \AA$ for the N1-carbazole and N3-carbazole units, respectively) and the attached phenyl rings are $55.54(6)$ and $43.46(7)^{\circ}$.

\section{Supramolecular features}

In the crystal, the two molecules are linked into [110] chains by $\mathrm{N}-\mathrm{H} \cdots \mathrm{O}$ and $\mathrm{N}-\mathrm{H} \cdots \mathrm{N}$ hydrogen bonds involving the carbazole $\mathrm{N}$ atom of one independent molecule and the nitro group of the other (Table 1), as shown in Fig. 2. In addition, $\pi-$ $\pi$ stacking interactions occur along the $c$-axis direction $[C g 1 \cdots C g 1(1-x,-y, 1-z)=3.3963(9) \AA$ and $C g 8 \cdots C g 8(1-x,-y,-z)=3.3982(10) \AA$ where $C g 1$ and $C g 8$

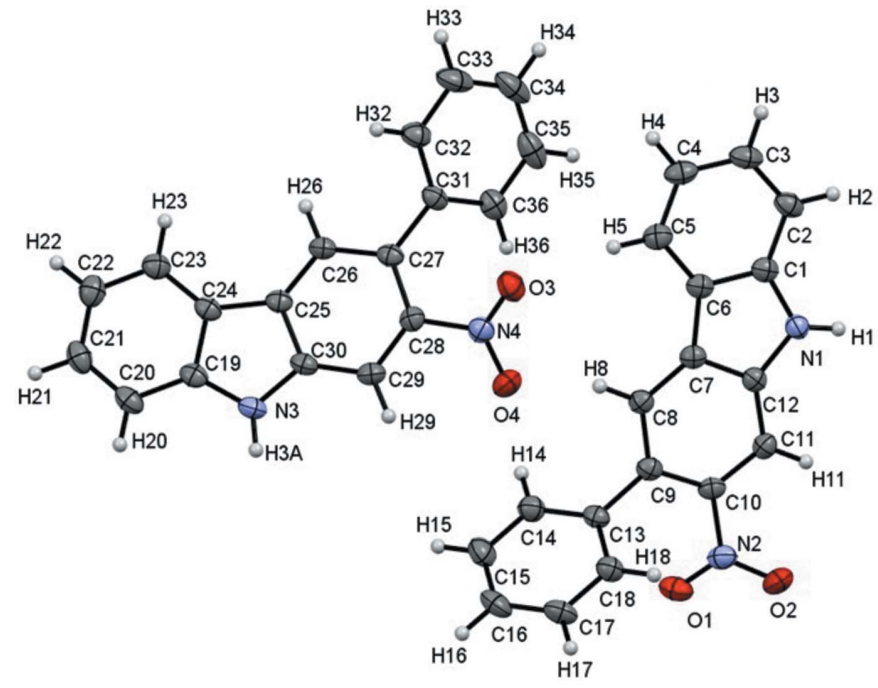

Figure 1

The asymmetric unit of the title compound, with atom labelling and displacement ellipsoids drawn at the $50 \%$ probability level.
Table 1

Hydrogen-bond geometry $\left(\AA{ }^{\circ}\right)$.

\begin{tabular}{lllll}
\hline$D-\mathrm{H} \cdots A$ & $D-\mathrm{H}$ & $\mathrm{H} \cdots A$ & $D \cdots A$ & $D-\mathrm{H} \cdots A$ \\
\hline $\mathrm{N} 1-\mathrm{H} 1 \cdots \mathrm{O} 3^{\mathrm{i}}$ & $0.88(1)$ & $2.33(1)$ & $3.1825(16)$ & $162(1)$ \\
$\mathrm{N} 1-\mathrm{H} 1 \cdots 4^{\mathrm{i}}$ & $0.88(1)$ & $2.38(1)$ & $3.1331(17)$ & $143(1)$ \\
$\mathrm{N} 1-\mathrm{H} 1 \cdots \mathrm{N}^{\mathrm{i}}$ & $0.88(1)$ & $2.59(1)$ & $3.4610(17)$ & $168(1)$ \\
$\mathrm{N} 3-\mathrm{H} 3 A \cdots \mathrm{O} 1^{\mathrm{ii}}$ & $0.88(1)$ & $2.26(1)$ & $3.1079(18)$ & $162(1)$ \\
$\mathrm{N} 3-\mathrm{H} 3 A \cdots \mathrm{O} 2^{\mathrm{ii}}$ & $0.88(1)$ & $2.45(1)$ & $3.2039(19)$ & $143(1)$ \\
$\mathrm{N} 3-\mathrm{H} 3 A \cdots 2^{\mathrm{ii}}$ & $0.88(1)$ & $2.60(1)$ & $3.4700(19)$ & $170(1)$ \\
\hline
\end{tabular}

Symmetry codes: (i) $-x+1,-y,-z+1$; (ii) $-x+2,-y+1,-z+1$.

are the centroids of the N1/C1/C6/C7/C12 and N3/C19/C24/ $\mathrm{C} 25 / \mathrm{C} 30$ rings, respectively] with adjacent carbazole rings within the stacks being almost parallel. The combination of hydrogen bonding and $\pi$-stacked carbazole ring systems results in the formation of a three-dimensional interaction.

\section{Database survey}

A search of the Cambridge Structural Database (CSD Version 5.42, November 2020; Groom et al. 2016) using a fragment composed of carbazole with a nitro group gave only one hit, which did not have much in common with the title compound. The most similar reported compound is ABEPON (9-ethyl-3methyl-1,6-dinitrocarbazole; Asker et al., 2004), whose main component consists of a nitro group on the carbazole ring. Examples of carbazole compounds substituted in the 3-position include ABAFOA (9- $p$-tolyl- $9 H$-carbazole-3-carbonitrile;

(a)
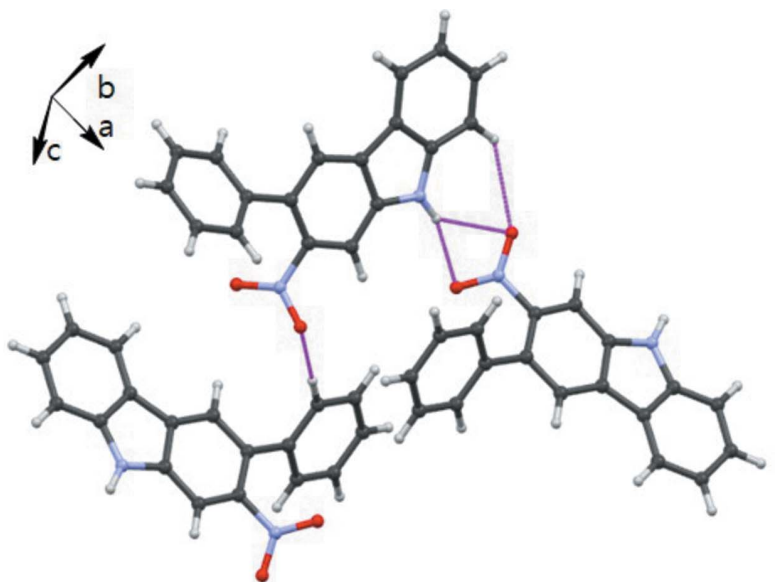

(b)

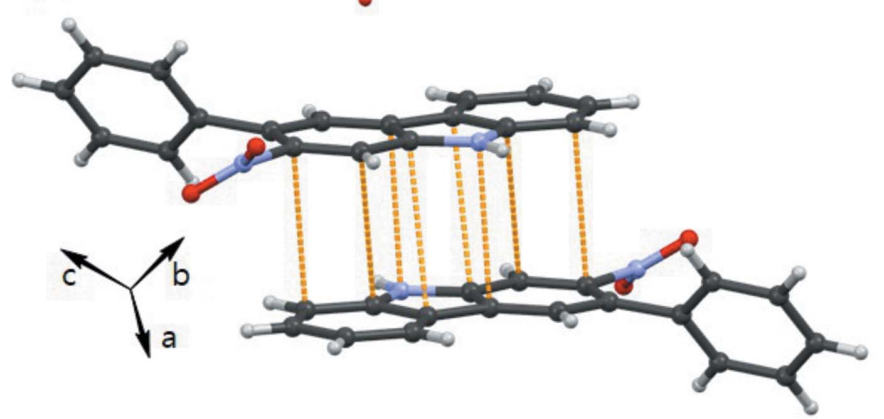

Figure 2

A plot showing $(a)$ the intermolecular $\mathrm{N}-\mathrm{H} \cdots \mathrm{O}, \mathrm{C}-\mathrm{H} \cdots \mathrm{O}$ hydrogen bonds and $(b) \pi-\pi$ interactions. 

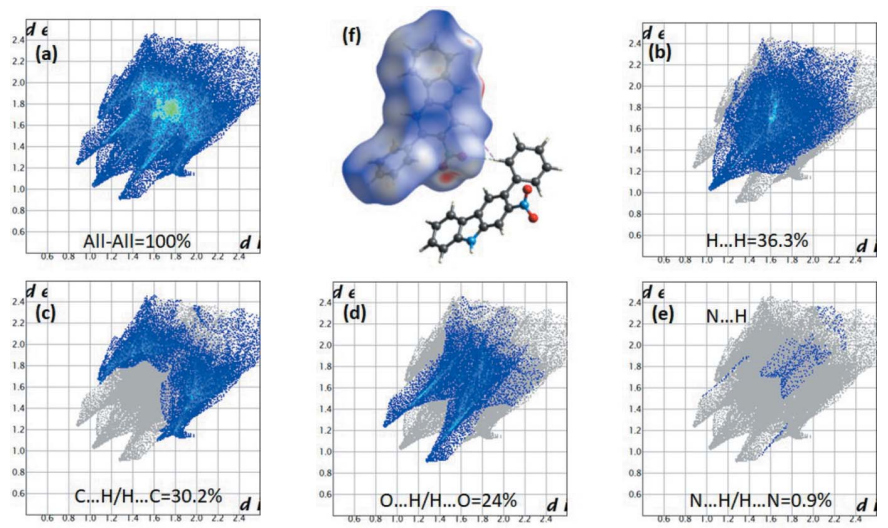

Figure 3

The Hirshfeld surface of the title compound mapped over $d_{\text {norm }}$ to visualize the intermolecular interactions.

Ramathilagam et al., 2011), ADALOH [3,6-dibromo-9-(4tolylsulfonyl)-9H-carbazole; Li et al., 2006], ANUWUD (dimethyl 9H-carbazole-1,3-dicarboxalate; Verma et al., 2015) and ATAWEZ [3,6-dimethoxy-9-(2-trifluoromethyl)phenyl9H-carbazole; Matsubara et al., 2016].

\section{Hirshfeld surface analysis}

A Hirshfeld surface analysis (McKinnon et al.,2007; Spackman \& Jayatilaka et al., 2009) of compound 1 was performed with CrystalExplorer17 (Turner et al., 2017) to give an insight into the intermolecular interactions. The Hirsfeld surface was calculated using a standard (high) surface resolution with the three-dimensional $d_{\text {norm }}$ surface plotted over a fixed colour scale of -0.1339 (red) to 1.4773 a.u. (blue) as shown in Fig. 3. The red spots indicate short contacts, i.e. negative $d_{\text {norm }}$ values on the surface, which highlight the hydrogen-bonding interactions.

The 2D finger plots shown in Fig. 3 indicate that the most important contributions to the overall surface are from $\mathrm{H} \cdots \mathrm{H}$ $(36.3 \%), \mathrm{C} \cdots \mathrm{H} / \mathrm{H} \cdots \mathrm{C}(30.2 \%)$ and $\mathrm{O} \cdots \mathrm{H} / \mathrm{H} \cdots \mathrm{O}(24 \%)$ interactions whereas the contribution of $\mathrm{N} \cdots \mathrm{H} / \mathrm{H} \cdots \mathrm{N}$ interactions is almost negligible at $0.9 \%$.
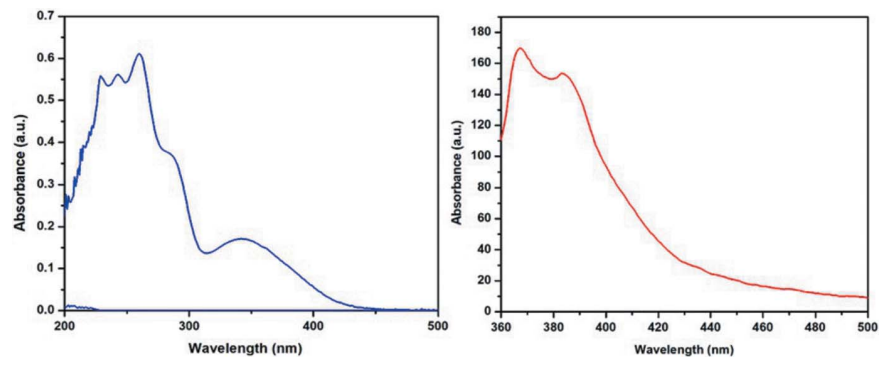

Figure 4

Absorption and emission spectra of the title compound $\mathbf{1}$ in DCM. The emission spectrum was excited at $350 \mathrm{~nm}$.

\section{Photophysical study}

The absorption and emission spectra of compound $\mathbf{1}$ were measured in dilute $\mathrm{CH}_{2} \mathrm{Cl}_{2}$ solution at room temperature, as shown in Fig. 4. Compound $\mathbf{1}$ exhibits an absorption band at $260 \mathrm{~nm}$ to $410 \mathrm{~nm}$, which can be assigned to the carbazole moieties. The broad absorption bands at the lower energy peak around $350 \mathrm{~nm}$ suggest the formation of the carbazole dimer excimer from the carbazole groups. The PL spectrum of compound 1 excited at $350 \mathrm{~nm}$ shows a dominant blue-violet broad peak at $400 \mathrm{~nm}$ associated with the emission from the carbazole excimer.

\section{Synthesis and crystallization}

The synthesis of the title compound is shown in Fig. 5. The reaction yielded single and double Cadogan ring-closure products. First we prepared dinitro compound a by a nitration reaction and then we synthesized terphenyl compound $\mathbf{b}$ by performing double Suzuki-coupling reaction on 1,5-dibromo2,4-dinitrobenzene and benzeneboronic acid. A two-necked flask fitted with a condenser was charged with 1,3-dinitro-4,6diphenyl benzene (b) $(0.320 \mathrm{~g}, 1 \mathrm{mmol})$ and $0.655 \mathrm{~g}$ $(2.5 \mathrm{mmol})$ of triphenylphosphine. $8 \mathrm{~mL}$ of the solvent $o$-dichlorobenzene were added $o$ the reaction mixture. The resulting reaction mixture was stirred at $473 \mathrm{~K}$ under nitrogen for $24 \mathrm{~h}$. The solvent was removed under reduced pressure at $333 \mathrm{~K}$ and the crude product was purified by column chromatography (silica gel, 10\% EA in hexanes as eluent) to provide $0.230 \mathrm{~g}$ of the title product as a beige solid (yield: $86 \%) .{ }^{1} \mathrm{H}$ NMR $(500 \mathrm{MHz}, \mathrm{CDCl} 3): \delta 8.39(s, 1 \mathrm{H}), 8.09(d, J=$ $8.2 \mathrm{~Hz}, 1 \mathrm{H}), 8.05(d, J=9.9 \mathrm{~Hz}, 2 \mathrm{H}), 7.56-7.51(m, 2 \mathrm{H}), 7.48-$ $7.38(m, 5 \mathrm{H}), 7.32(d d d, J=8.0,6.4,1.7 \mathrm{~Hz}, 1 \mathrm{H})$.

\section{Refinement}

Crystal data, data collection and structure refinement details are summarized in Table 2. N-bound $\mathrm{H}$ atoms were refined with $U_{\text {iso }}(\mathrm{H})=1.2 U_{\text {eq }}(\mathrm{N})$. C-bound $\mathrm{H}$ atoms were positioned
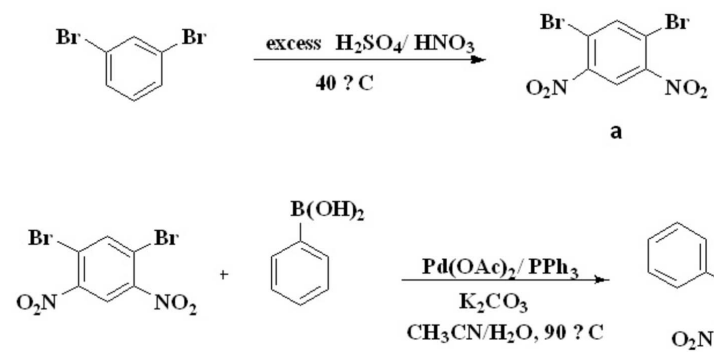<smiles>O=[N+]([O-])c1cc([N+](=O)[O-])c(-c2ccccc2)cc1-c1ccccc1</smiles><smiles>O=[N+]([O-])c1cc([N+](=O)[O-])c(-c2ccccc2)cc1-c1ccccc1</smiles>

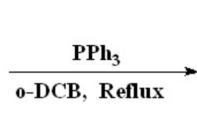<smiles>O=[N+]([O-])c1cc2[nH]c3ccccc3c2cc1-c1ccccc1</smiles>

1

Figure 5

Reaction scheme. 
Table 2

Experimental details.

\begin{tabular}{|c|c|}
\hline \multicolumn{2}{|l|}{ Crystal data } \\
\hline Chemical formula & $\mathrm{C}_{18} \mathrm{H}_{12} \mathrm{~N}_{2} \mathrm{O}_{2}$ \\
\hline$M_{\mathrm{r}}$ & 288.30 \\
\hline Crystal system, space group & Triclinic, $P \overline{1}$ \\
\hline Temperature (K) & 100 \\
\hline$a, b, c(\AA)$ & $9.2660(3), 12.9590(4), 13.1010(4)$ \\
\hline$\alpha, \beta, \gamma\left({ }^{\circ}\right)$ & $\begin{array}{l}96.2487(15), 109.1813(15) \\
\quad 106.1061(14)\end{array}$ \\
\hline$V\left(\AA^{3}\right)$ & $1392.39(8)$ \\
\hline$Z$ & 4 \\
\hline Radiation type & Мo $K \alpha$ \\
\hline$\mu\left(\mathrm{mm}^{-1}\right)$ & 0.09 \\
\hline Crystal size $(\mathrm{mm})$ & $0.1 \times 0.1 \times 0.1$ \\
\hline \multicolumn{2}{|l|}{ Data collection } \\
\hline Diffractometer & Bruker APEXII CCD \\
\hline Absorption correction & $\begin{array}{l}\text { Multi-scan (SADABS; Bruker, } \\
\text { 2014) }\end{array}$ \\
\hline$T_{\min }, T_{\max }$ & $0.628,0.745$ \\
\hline $\begin{array}{l}\text { No. of measured, independent and } \\
\text { observed }[I>2 \sigma(I)] \text { reflections }\end{array}$ & $17357,5277,4470$ \\
\hline$R_{\text {int }}$ & 0.026 \\
\hline$(\sin \theta / \lambda)_{\max }\left(\AA^{-1}\right)$ & 0.611 \\
\hline \multicolumn{2}{|l|}{ Refinement } \\
\hline$R\left[F^{2}>2 \sigma\left(F^{2}\right)\right], w R\left(F^{2}\right), S$ & $0.041,0.115,1.08$ \\
\hline No. of reflections & 5277 \\
\hline No. of parameters & 398 \\
\hline $\mathrm{H}$-atom treatment & $\mathrm{H}$-atom parameters constrained \\
\hline$\Delta \rho_{\max }, \Delta \rho_{\min }\left(\mathrm{e} \AA^{-3}\right)$ & $0.40,-0.31$ \\
\hline
\end{tabular}

Computer programs: APEX2 and SAINT (Bruker, 2014), SHELXT (Sheldrick, 2015a), SHELXL (Sheldrick, 2015b) and OLEX2 (Dolomanov et al., 2009).

geometrically $(\mathrm{C}-\mathrm{H}=0.95 \AA)$ and refined as riding with $U_{\text {iso }}(\mathrm{H})=1.2 U_{\text {eq }}(\mathrm{C})$.

\section{Funding information}

Funding for this research was provided by: National Research Foundation of Korea (grant No. 2019R1A2C1001989).

\section{References}

Asker, E. \& Masnovi, J. (2004). Acta Cryst. E60, o1613-o1615.

Bao, L., Zhu, J., Song, W., Zhou, H., Huang, J., Mu, H. \& Su, J. (2020). Org. Electron. 83, 105672.

Bashir, M., Bano, A., Ijaz, A. S. \& Chaudhary, B. A. (2015). Molecules, 20, 13496-13517.

Bruker (2014). APEX2 SAINT and SADABS. Bruker AXS Inc., Madison, Wisconsin, USA.

Chan, L.-H., Lin, L.-C., Yao, C.-H., Liu, Y.-R., Jiang, Z.-J. \& Cho, T.-Y. (2013). Thin Solid Films, 544, 386-391.

Chen, C.-H., Wang, Y., Michinobu, T., Chang, S.-W., Chiu, Y.-C., Ke, C.-Y. \& Liou, G.-S. (2020). Appl. Mater. Interfaces, 12, 6144-6150.

Collin, G., Höke, H. \& Talbiersky, J. (2006). Carbazole. In Ullmann's Encyclopedia of Industrial Chemistry. Weinheim: Wiley-VCH.

Dolomanov, O. V., Bourhis, L. J., Gildea, R. J., Howard, J. A. K. \& Puschmann, H. (2009). J. Appl. Cryst. 42, 339-341.

Groom, C. R., Bruno, I. J., Lightfoot, M. P. \& Ward, S. C. (2016). Acta Cryst. B72, 171-179.
Koli, M. R., Labiod, A., Chakraborty, S., Kumar, M., Lévêque, P., Ulrich, G., Leclerc, N., Jacquemin, D. \& Mula, S. (2020). ChemPhotoChem 4, 729-741.

Li, M., Wang, Z., Liang, M., Liu, L., Wang, X., Sun, Z. \& Xue, S. (2018). J. Phys. Chem. C, 122, 24014-24024.

Li, N., Huang, P.-M., Xiong, X.-L., Xu, X.-D. \& Shao, Z.-J. (2006). Acta Cryst. E62, o1805-o1806.

Lokhande, P. K. M., Sonigara, K. K., Jadhav, M. M., Patil, D. S., Soni, S. S. \& Sekar, N. (2019). ChemistrySelect 4, 4044-4056.

Matsubara, R., Shimada, T., Kobori, Y., Yabuta, T., Osakai, T. \& Hayashi, M. (2016). Chem. Asian J. 11, 2006-2010.

McKinnon, J. J., Jayatilaka, D. \& Spackman, M. A. (2007). Chem. Commun. pp. 3814-3816.

Nandy, B. C., Gupta, A., Mittal, A. \& Vyas, V. (2014). J. Biomed. Pharm. Res, 3, 42-48.

Niu, P., Huang, H., Zhao, L., Zhang, C., Shen, Z. \& Li, M. (2021). J. Electroanal. Chem. 894, 115352.

Oda, S., Kumano, W., Hama, T., Kawasumi, R., Yoshiura, K. \& Hatakeyama, T. (2021). Angew. Chem. 133, 2918-2922.

Ramathilagam, C., Venkatesan, N., Rajakumar, P., Umarani, P. R. \& Manivannan, V. (2011). Acta Cryst. E67, o2796.

Reig, M., Puigdollers, J. \& Velasco, D. (2015). J. Mater. Chem. C. 3, 506-513.

Sheldrick, G. M. (2015a). Acta Cryst. A71, 3-8.

Sheldrick, G. M. (2015b). Acta Cryst. C71, 3-8.

Spackman, M. A. \& Jayatilaka, D. (2009). CrystEngComm, 11, 19-32.

Srivastava, A. \& Chakrabarti, P. (2017). Appl. Phys. A, 123, 784.

Sun, D., Ren, Z., Bryce, M. R. \& Yan, S. (2015). J. Mater. Chem. C. 3, 9496-9508.

Sutanto, A. A., Joseph, V., Igci, C., Syzgantseva, O. A., Syzgantseva, M. A., Jankauskas, V., Rakstys, K., Queloz, V. I., Huang, P.-Y., Ni, J.-S., Kinge, S., Asiri, A. M., Chen, M. \& Nazeeruddin, M. K. (2021). Chem. Mater. 33, 3286-3296.

Svetlichnyi, V. M., Alexandrova, E. L., Miagkova, L. A., Matushina, N. V., Nekrasova, T. N., Tameev, A. R., Stepanenko, S. N., Vannikov, A. V. \& Kudryavtsev, V. V. (2010). Semiconductors, 44, 1581-1587.

Szafraniec-Gorol, G., Slodek, A., Zych, D., Vasylieva, M., Siwy, M., Sulowska, K., Maćkowski, S., Taydakov, I., Goriachiy, D. \& SchabBalcerzak, E. (2021). J. Mater. Chem. C. 9, 7351-7362.

Turner, M. J., McKinnon, J. J., Wolff, S. K., Grimwood, D. J., Spackman, P. R., Jayatilaka, D. \& Spackman, M. A. (2017). CrystalExplorer17. University of Western Australia. http://hirshfeldsurface. net

Verma, A. K., Danodia, A. K., Saunthwal, R. K., Patel, M. \& Choudhary, D. (2015). Org. Lett. 17, 3658-3661.

Wakim, S., Aïch, B. R., Tao, Y. \& Leclerc, M. (2008). Polym. Rev. 48, 432-462.

Xiang, S., Lv, X., Sun, S., Zhang, Q., Huang, Z., Guo, R., Gu, H., Liu, S. \& Wang, L. (2018). J. Mater. Chem. C. 6, 5812-5820.

Yang, J., Devillers, C. H., Fleurat-Lessard, P., Jiang, H., Wang, S., Gros, C. P., Gupta, G., Sharma, G. D. \& Xu, H. (2020). Dalton Trans. 49, 5606-5617.

Yavuz, Ö., Sezer, E. \& Saraç, A. S. (2001). Polym. Int. 50, 271-276.

Zhang, D., Song, X., Cai, M., Kaji, H. \& Duan, L. (2018). Adv. Mater. 30, 1705406

Zhang, X.-H., Wang, Z.-S., Cui, Y., Koumura, N., Furube, A. \& Hara, K. (2009). J. Phys. Chem. C, 113, 13409-13415.

Zhou, H., Yin, M., Zhao, Z., Miao, Y., Jin, X., Huang, J., Gao, Z., Wang, H., Su, J. \& Tian, H. (2021). J. Mater. Chem. C. 9, 5899-5907. 


\section{supporting information}

Acta Cryst. (2021). E77, 887-890 [https://doi.org/10.1107/S2056989021007726]

\section{Crystal structure, Hirshfeld surface and photophysical analysis of 2-nitro-3- phenyl-9H-carbazole}

Neetu Singh, Ga Hee Noh, Hyoung-Ryun Park and Junseong Lee

Computing details

Data collection: APEX2 (Bruker, 2014); cell refinement: SAINT (Bruker, 2014); data reduction: SAINT (Bruker, 2014); program(s) used to solve structure: SHELXT (Sheldrick, 2015a); program(s) used to refine structure: SHELXL (Sheldrick, 2015b); molecular graphics: OLEX2 (Dolomanov et al., 2009); software used to prepare material for publication: OLEX2 (Dolomanov et al., 2009).

2-Nitro-3-phenyl-9H-carbazole

Crystal data

$\mathrm{C}_{18} \mathrm{H}_{12} \mathrm{~N}_{2} \mathrm{O}_{2}$

$M_{r}=288.30$

Triclinic, $P \overline{1}$

$a=9.2660(3) \AA$

$b=12.9590(4) \AA$

$c=13.1010(4) \AA$

$\alpha=96.2487(15)^{\circ}$

$\beta=109.1813(15)^{\circ}$

$\gamma=106.1061(14)^{\circ}$

$V=1392.39(8) \AA^{3}$

$Z=4$

$F(000)=600$

$D_{\mathrm{x}}=1.375 \mathrm{Mg} \mathrm{m}^{-3}$

Mo $K \alpha$ radiation, $\lambda=0.71073 \AA$

Cell parameters from 8081 reflections

$\theta=2.6-25.7^{\circ}$

$\mu=0.09 \mathrm{~mm}^{-1}$

$T=100 \mathrm{~K}$

Block, white

Data collection

Bruker APEXII CCD

$0.1 \times 0.1 \times 0.1 \mathrm{~mm}$

diffractometer

$\varphi$ and $\omega$ scans

Absorption correction: multi-scan

(SADABS; Bruker, 2014)

$T_{\text {min }}=0.628, T_{\max }=0.745$

17357 measured reflections

5277 independent reflections

4470 reflections with $I>2 \sigma(I)$

$R_{\text {int }}=0.026$

$\theta_{\max }=25.7^{\circ}, \theta_{\min }=1.7^{\circ}$

$h=-11 \rightarrow 11$

$k=-15 \rightarrow 15$

$l=-15 \rightarrow 15$

\section{Refinement}

Refinement on $F^{2}$

Least-squares matrix: full

$R\left[F^{2}>2 \sigma\left(F^{2}\right)\right]=0.041$

$w R\left(F^{2}\right)=0.115$

$S=1.08$

5277 reflections

398 parameters

0 restraints

Hydrogen site location: inferred from neighbouring sites

$\mathrm{H}$-atom parameters constrained

$w=1 /\left[\sigma^{2}\left(F_{\mathrm{o}}{ }^{2}\right)+(0.0657 P)^{2}+0.2853 P\right]$

where $P=\left(F_{\mathrm{o}}^{2}+2 F_{\mathrm{c}}^{2}\right) / 3$

$(\Delta / \sigma)_{\max }=0.001$

$\Delta \rho_{\max }=0.40 \mathrm{e} \AA^{-3}$

$\Delta \rho_{\min }=-0.31$ e $\AA^{-3}$

Extinction correction: SHELXL, $F c^{*}=\mathrm{kFc}\left[1+0.001 \mathrm{xFc}^{2} \lambda^{3} / \sin (2 \theta)\right]^{-1 / 4}$

Extinction coefficient: 0.026 (3) 


\section{Special details}

Geometry. All esds (except the esd in the dihedral angle between two 1.s. planes) are estimated using the full covariance matrix. The cell esds are taken into account individually in the estimation of esds in distances, angles and torsion angles; correlations between esds in cell parameters are only used when they are defined by crystal symmetry. An approximate (isotropic) treatment of cell esds is used for estimating esds involving l.s. planes.

Fractional atomic coordinates and isotropic or equivalent isotropic displacement parameters $\left(\AA^{2}\right)$

\begin{tabular}{|c|c|c|c|c|}
\hline & $x$ & $y$ & $z$ & $U_{\text {iso }} * / U_{\text {eq }}$ \\
\hline $\mathrm{O} 1$ & $1.07827(13)$ & $0.32279(9)$ & $0.71767(9)$ & 0.0379 \\
\hline $\mathrm{O} 2$ & $1.11420(15)$ & $0.18678(11)$ & $0.79164(10)$ & 0.0523 \\
\hline N1 & $0.58804(14)$ & $-0.11853(9)$ & $0.53169(10)$ & $0.0267(3)$ \\
\hline H1 & 0.5808 & -0.1487 & 0.5876 & $0.032^{*}$ \\
\hline N2 & $1.04678(14)$ & $0.22353(11)$ & $0.71390(10)$ & $0.0324(3)$ \\
\hline $\mathrm{C} 1$ & $0.48711(16)$ & $-0.16207(11)$ & $0.42177(12)$ & 0.0258 \\
\hline $\mathrm{C} 2$ & $0.35659(17)$ & $-0.26036(11)$ & $0.37438(13)$ & $0.0312(3)$ \\
\hline $\mathrm{H} 2$ & 0.3235 & -0.3083 & 0.4185 & $0.037^{*}$ \\
\hline $\mathrm{C} 3$ & $0.27777(17)$ & $-0.28488(12)$ & $0.26075(13)$ & $0.0339(3)$ \\
\hline H3 & 0.1894 & -0.3517 & 0.2261 & $0.041^{*}$ \\
\hline $\mathrm{C} 4$ & $0.32410(17)$ & $-0.21428(12)$ & $0.19502(13)$ & 0.0338 \\
\hline $\mathrm{H} 4$ & 0.2663 & -0.2334 & 0.1171 & $0.041^{*}$ \\
\hline $\mathrm{C} 5$ & $0.45313(17)$ & $-0.11701(12)$ & $0.24236(12)$ & $0.0306(3)$ \\
\hline H5 & 0.4844 & -0.0691 & 0.1977 & $0.037^{*}$ \\
\hline C6 & $0.53657(16)$ & -0.09055 (11) & $0.35684(11)$ & 0.0253 \\
\hline $\mathrm{C} 7$ & $0.67397(15)$ & $0.00101(11)$ & $0.43280(11)$ & $0.0241(3)$ \\
\hline $\mathrm{C} 8$ & $0.77372(16)$ & 0.09677 (11) & $0.41936(11)$ & $0.0250(3)$ \\
\hline H8 & 0.7534 & 0.1117 & 0.3475 & $0.030^{*}$ \\
\hline C9 & $0.90234(16)$ & $0.17073(11)$ & $0.50954(11)$ & 0.0248 \\
\hline $\mathrm{C} 10$ & $0.92260(16)$ & $0.14514(11)$ & $0.61431(11)$ & $0.0257(3)$ \\
\hline $\mathrm{C} 11$ & $0.82673(16)$ & $0.05185(11)$ & $0.63224(11)$ & $0.0263(3)$ \\
\hline H11 & 0.8457 & 0.0380 & 0.7044 & $0.032^{*}$ \\
\hline $\mathrm{C} 12$ & $0.70141(16)$ & -0.02055 & $0.53961(11)$ & $0.0240(3)$ \\
\hline $\mathrm{C} 13$ & $1.01868(16)$ & $0.26510(11)$ & $0.49125(11)$ & 0.0255 \\
\hline $\mathrm{C} 14$ & $0.96555(18)$ & $0.33925(12)$ & $0.43259(12)$ & $0.0314(3)$ \\
\hline H14 & 0.8537 & 0.3314 & 0.4059 & $0.038^{*}$ \\
\hline $\mathrm{C} 15$ & $1.0741(2)$ & $0.42439(12)$ & $0.41272(14)$ & $0.0377(4)$ \\
\hline H15 & 1.0363 & 0.4747 & 0.3728 & $0.045^{*}$ \\
\hline C16 & $1.2368(2)$ & $0.43656(12)$ & $0.45059(14)$ & $0.0389(4)$ \\
\hline H16 & 1.3110 & 0.4949 & 0.4366 & $0.047^{*}$ \\
\hline $\mathrm{C} 17$ & $1.29147(18)$ & $0.36317(12)$ & $0.50921(13)$ & $0.0355(4)$ \\
\hline H17 & 1.4035 & 0.3716 & 0.5359 & $0.043^{*}$ \\
\hline C18 & $1.18363(17)$ & $0.27788(12)$ & $0.52902(12)$ & $0.0297(3)$ \\
\hline H18 & 1.2219 & 0.2275 & 0.5686 & $0.036^{*}$ \\
\hline $\mathrm{O} 3$ & $0.39259(13)$ & $0.17339(8)$ & $0.23420(9)$ & $0.0372(3)$ \\
\hline $\mathrm{O} 4$ & $0.54564(13)$ & $0.31480(10)$ & $0.36730(9)$ & $0.0424(3)$ \\
\hline N3 & $0.55743(14)$ & $0.60781(9)$ & $0.13124(10)$ & $0.0289(3)$ \\
\hline $\mathrm{H} 3 \mathrm{~A}$ & 0.6607 & 0.6425 & 0.1717 & $0.035^{*}$ \\
\hline N4 & $0.44465(14)$ & $0.27322(10)$ & $0.27258(10)$ & $0.0302(3)$ \\
\hline
\end{tabular}




$\begin{array}{lllll}\text { C19 } & 0.46298(17) & 0.64286(11) & 0.04645(12) & 0.0273(3) \\ \text { C20 } & 0.50540(19) & 0.73722(11) & 0.00593(13) & 0.0320(3) \\ \text { H20 } & 0.6134 & 0.7872 & 0.0348 & 0.038^{*} \\ \text { C21 } & 0.3850(2) & 0.75518(12) & -0.07728(13) & 0.0345(4) \\ \text { H21 } & 0.4103 & 0.8199 & -0.1050 & 0.041^{*} \\ \text { C22 } & 0.22615(19) & 0.68083(12) & -0.12246(13) & 0.0345(3) \\ \text { H22 } & 0.1460 & 0.6956 & -0.1802 & 0.041^{*} \\ \text { C23 } & 0.18495(18) & 0.58607(12) & -0.08380(12) & 0.0307(3) \\ \text { H23 } & 0.0776 & 0.5351 & -0.1153 & 0.037^{*} \\ \text { C24 } & 0.30359(16) & 0.56668(11) & 0.00213(12) & 0.0263(3) \\ \text { C25 } & 0.30496(16) & 0.48076(11) & 0.06358(11) & 0.0250(3) \\ \text { C26 } & 0.18845(16) & 0.38326(11) & 0.05811(11) & 0.0253(3) \\ \text { H26 } & 0.0807 & 0.3646 & 0.0062 & 0.030^{*} \\ \text { C27 } & 0.22709(16) & 0.31273(11) & 0.12733(11) & 0.0248(3) \\ \text { C28 } & 0.38882(16) & 0.34634(11) & 0.20367(11) & 0.0255(3) \\ \text { C29 } & 0.50785(16) & 0.44388(11) & 0.21468(11) & 0.0269(3) \\ \text { H29 } & 0.6141 & 0.4642 & 0.2692 & 0.032^{*} \\ \text { C30 } & 0.46465(16) & 0.51022(11) & 0.14253(11) & 0.0254(3) \\ \text { C31 } & 0.09599(16) & 0.21235(11) & 0.12135(12) & 0.0260(3) \\ \text { C32 } & -0.01531(18) & 0.14815(11) & 0.01888(13) & 0.0328(3) \\ \text { H32 } & -0.0024 & 0.1655 & -0.0469 & 0.039^{*} \\ \text { C33 } & -0.1454(2) & 0.05885(12) & 0.01173(16) & 0.0446(4) \\ \text { H33 } & -0.2219 & 0.0162 & -0.0587 & 0.054^{*} \\ \text { C34 } & -0.1634(2) & 0.03214(13) & 0.10686(18) & 0.0467(5) \\ \text { H34 } & -0.2524 & -0.0288 & 0.1021 & 0.056^{*} \\ \text { C35 } & -0.0517(2) & 0.09420(13) & 0.20895(16) & 0.0413(4) \\ \text { H35 } & -0.0628 & 0.0746 & 0.2745 & 0.050^{*} \\ \text { C36 } & 0.07586(18) & 0.18444(12) & 0.21679(13) & 0.0319(3) \\ \text { H36 } & 0.1503 & 0.2278 & 0.2876 & 0.038^{*} \\ & & & & \end{array}$

Atomic displacement parameters $\left(\AA^{2}\right)$

\begin{tabular}{lllllll}
\hline & $U^{11}$ & $U^{22}$ & $U^{33}$ & $U^{12}$ & $U^{13}$ & $U^{23}$ \\
\hline $\mathrm{O} 1$ & $0.0334(6)$ & $0.0313(6)$ & $0.0382(6)$ & $0.0010(5)$ & $0.0118(5)$ & $-0.0041(5)$ \\
$\mathrm{O} 2$ & $0.0430(7)$ & $0.0552(8)$ & $0.0326(6)$ & $-0.0003(6)$ & $-0.0058(5)$ & $0.0137(5)$ \\
$\mathrm{N} 1$ & $0.0263(6)$ & $0.0246(6)$ & $0.0297(6)$ & $0.0080(5)$ & $0.0110(5)$ & $0.0083(5)$ \\
$\mathrm{N} 2$ & $0.0245(6)$ & $0.0380(7)$ & $0.0276(7)$ & $0.0035(5)$ & $0.0081(5)$ & $0.0029(5)$ \\
$\mathrm{C} 1$ & $0.0225(6)$ & $0.0235(7)$ & $0.0321(8)$ & $0.0106(5)$ & $0.0095(6)$ & $0.0029(5)$ \\
$\mathrm{C} 2$ & $0.0268(7)$ & $0.0229(7)$ & $0.0428(9)$ & $0.0080(6)$ & $0.0129(7)$ & $0.0047(6)$ \\
$\mathrm{C} 3$ & $0.0251(7)$ & $0.0255(7)$ & $0.0429(9)$ & $0.0057(6)$ & $0.0084(7)$ & $-0.0036(6)$ \\
$\mathrm{C} 4$ & $0.0282(7)$ & $0.0353(8)$ & $0.0307(8)$ & $0.0097(6)$ & $0.0067(6)$ & $-0.0046(6)$ \\
$\mathrm{C} 5$ & $0.0287(7)$ & $0.0330(8)$ & $0.0290(8)$ & $0.0098(6)$ & $0.0113(6)$ & $0.0023(6)$ \\
$\mathrm{C} 6$ & $0.0224(7)$ & $0.0242(7)$ & $0.0292(7)$ & $0.0090(5)$ & $0.0099(6)$ & $0.0021(5)$ \\
$\mathrm{C} 7$ & $0.0214(6)$ & $0.0257(7)$ & $0.0259(7)$ & $0.0089(5)$ & $0.0097(6)$ & $0.0032(5)$ \\
C8 & $0.0231(7)$ & $0.0276(7)$ & $0.0253(7)$ & $0.0088(5)$ & $0.0101(6)$ & $0.0058(5)$ \\
C9 & $0.0214(6)$ & $0.0251(7)$ & $0.0288(7)$ & $0.0086(5)$ & $0.0102(6)$ & $0.0048(5)$ \\
C10 & $0.0204(6)$ & $0.0280(7)$ & $0.0258(7)$ & $0.0071(5)$ & $0.0068(6)$ & $0.0022(5)$ \\
C11 & $0.0254(7)$ & $0.0300(7)$ & $0.0245(7)$ & $0.0109(6)$ & $0.0091(6)$ & $0.0069(6)$
\end{tabular}




\begin{tabular}{|c|c|c|c|c|c|c|}
\hline $\mathrm{C} 12$ & $0.0220(6)$ & $0.0229(6)$ & $0.0296(7)$ & $0.0096(5)$ & $0.0109(6)$ & $0.0058(5)$ \\
\hline $\mathrm{C} 13$ & $0.0244(7)$ & $0.0233(7)$ & $0.0266(7)$ & $0.0051(5)$ & $0.0104(6)$ & $0.0013(5)$ \\
\hline $\mathrm{C} 14$ & $0.0280(7)$ & $0.0295(7)$ & $0.0344(8)$ & $0.0087(6)$ & $0.0099(6)$ & $0.0057(6)$ \\
\hline C15 & $0.0440(9)$ & $0.0245(7)$ & $0.0432(9)$ & $0.0093(7)$ & $0.0159(8)$ & $0.0088(6)$ \\
\hline $\mathrm{C} 16$ & $0.0394(9)$ & $0.0221(7)$ & $0.0489(10)$ & $-0.0014(6)$ & $0.0201(8)$ & $0.0025(6)$ \\
\hline $\mathrm{C} 17$ & $0.0251(7)$ & $0.0303(8)$ & $0.0446(9)$ & $0.0017(6)$ & $0.0134(7)$ & $0.0005(6)$ \\
\hline C18 & $0.0264(7)$ & $0.0273(7)$ & $0.0337(8)$ & $0.0078(6)$ & $0.0111(6)$ & $0.0043(6)$ \\
\hline $\mathrm{O} 3$ & $0.0402(6)$ & $0.0291(6)$ & $0.0455(7)$ & $0.0135(5)$ & $0.0174(5)$ & $0.0120(5)$ \\
\hline O4 & $0.0354(6)$ & $0.0493(7)$ & $0.0313(6)$ & $0.0086(5)$ & $0.0023(5)$ & $0.0123(5)$ \\
\hline N3 & $0.0229(6)$ & $0.0228(6)$ & $0.0330(7)$ & $0.0011(5)$ & $0.0077(5)$ & $0.0008(5)$ \\
\hline N4 & $0.0258(6)$ & $0.0343(7)$ & $0.0316(7)$ & $0.0090(5)$ & $0.0122(5)$ & $0.0096(5)$ \\
\hline C19 & $0.0285(7)$ & $0.0231(7)$ & $0.0295(7)$ & $0.0068(6)$ & $0.0131(6)$ & $0.0003(5)$ \\
\hline $\mathrm{C} 20$ & $0.0357(8)$ & $0.0222(7)$ & $0.0390(8)$ & $0.0057(6)$ & $0.0196(7)$ & $0.0026(6)$ \\
\hline $\mathrm{C} 21$ & $0.0465(9)$ & $0.0258(7)$ & $0.0399(9)$ & $0.0143(7)$ & $0.0244(7)$ & $0.0099(6)$ \\
\hline $\mathrm{C} 22$ & $0.0418(9)$ & $0.0341(8)$ & $0.0337(8)$ & $0.0191(7)$ & $0.0159(7)$ & $0.0092(6)$ \\
\hline $\mathrm{C} 23$ & $0.0295(7)$ & 0.0288 (7) & $0.0328(8)$ & $0.0098(6)$ & $0.0114(6)$ & $0.0039(6)$ \\
\hline $\mathrm{C} 24$ & $0.0270(7)$ & $0.0217(7)$ & $0.0300(7)$ & $0.0069(5)$ & $0.0129(6)$ & $0.0016(5)$ \\
\hline $\mathrm{C} 25$ & $0.0244(7)$ & $0.0229(7)$ & $0.0256(7)$ & $0.0069(5)$ & $0.0094(6)$ & $0.0004(5)$ \\
\hline $\mathrm{C} 26$ & $0.0211(6)$ & $0.0239(7)$ & $0.0264(7)$ & $0.0046(5)$ & $0.0072(6)$ & $0.0013(5)$ \\
\hline $\mathrm{C} 27$ & $0.0243(7)$ & $0.0233(7)$ & $0.0249(7)$ & $0.0056(5)$ & $0.0102(6)$ & $0.0006(5)$ \\
\hline $\mathrm{C} 28$ & $0.0261(7)$ & $0.0268(7)$ & $0.0243(7)$ & $0.0091(6)$ & $0.0104(6)$ & $0.0047(5)$ \\
\hline $\mathrm{C} 29$ & $0.0220(7)$ & $0.0278(7)$ & $0.0254(7)$ & $0.0057(6)$ & $0.0059(6)$ & $0.0001(5)$ \\
\hline $\mathrm{C} 30$ & $0.0231(7)$ & $0.0219(6)$ & $0.0279(7)$ & $0.0036(5)$ & $0.0104(6)$ & $-0.0004(5)$ \\
\hline $\mathrm{C} 31$ & $0.0237(7)$ & $0.0211(6)$ & $0.0346(8)$ & $0.0080(5)$ & $0.0126(6)$ & $0.0051(5)$ \\
\hline $\mathrm{C} 32$ & $0.0307(8)$ & $0.0245(7)$ & $0.0386(8)$ & $0.0079(6)$ & $0.0101(7)$ & $0.0019(6)$ \\
\hline $\mathrm{C} 33$ & $0.0321(8)$ & $0.0230(8)$ & $0.0640(12)$ & $0.0033(6)$ & $0.0085(8)$ & $-0.0033(7)$ \\
\hline C34 & $0.0356(9)$ & $0.0224(8)$ & $0.0860(14)$ & $0.0069(7)$ & $0.0295(10)$ & $0.0132(8)$ \\
\hline $\mathrm{C} 35$ & $0.0458(9)$ & $0.0322(8)$ & $0.0674(12)$ & $0.0200(7)$ & $0.0387(9)$ & $0.0226(8)$ \\
\hline $\mathrm{C} 36$ & $0.0335(8)$ & $0.0287(7)$ & $0.0408(9)$ & $0.0136(6)$ & $0.0199(7)$ & $0.0097(6)$ \\
\hline
\end{tabular}

Geometric parameters $\left(\AA,{ }^{\circ}\right)$

\begin{tabular}{llll}
\hline $\mathrm{O} 1-\mathrm{N} 2$ & $1.2291(17)$ & $\mathrm{O} 3-\mathrm{N} 4$ & $1.2296(16)$ \\
$\mathrm{O} 2-\mathrm{N} 2$ & $1.2275(17)$ & $\mathrm{O} 4-\mathrm{N} 4$ & $1.2319(16)$ \\
$\mathrm{N} 1-\mathrm{H} 1$ & 0.8800 & $\mathrm{~N} 3-\mathrm{H} 3 \mathrm{~A}$ & 0.8800 \\
$\mathrm{~N} 1-\mathrm{C} 1$ & $1.3829(18)$ & $\mathrm{N} 3-\mathrm{C} 19$ & $1.3776(19)$ \\
$\mathrm{N} 1-\mathrm{C} 12$ & $1.3734(17)$ & $\mathrm{N} 3-\mathrm{C} 30$ & $1.3716(17)$ \\
$\mathrm{N} 2-\mathrm{C} 10$ & $1.4610(18)$ & $\mathrm{N} 4-\mathrm{C} 28$ & $1.4600(18)$ \\
$\mathrm{C} 1-\mathrm{C} 2$ & $1.3966(19)$ & $\mathrm{C} 19-\mathrm{C} 20$ & $1.394(2)$ \\
$\mathrm{C} 1-\mathrm{C} 6$ & $1.409(2)$ & $\mathrm{C} 19-\mathrm{C} 24$ & $1.4128(19)$ \\
$\mathrm{C} 2-\mathrm{H} 2$ & 0.9500 & $\mathrm{C} 20-\mathrm{H} 20$ & 0.9500 \\
$\mathrm{C} 2-\mathrm{C} 3$ & $1.380(2)$ & $\mathrm{C} 20-\mathrm{C} 21$ & $1.374(2)$ \\
$\mathrm{C} 3-\mathrm{H} 3$ & 0.9500 & $\mathrm{C} 21-\mathrm{H} 21$ & 0.9500 \\
$\mathrm{C} 3-\mathrm{C} 4$ & $1.400(2)$ & $\mathrm{C} 21-\mathrm{C} 22$ & $1.401(2)$ \\
$\mathrm{C} 4-\mathrm{H} 4$ & 0.9500 & $\mathrm{C} 22-\mathrm{H} 22$ & 0.9500 \\
$\mathrm{C} 4-\mathrm{C} 5$ & $1.382(2)$ & $\mathrm{C} 22-\mathrm{C} 23$ & $1.383(2)$ \\
$\mathrm{C} 5-\mathrm{H} 5$ & 0.9500 & $\mathrm{C} 23-\mathrm{H} 23$ & 0.9500 \\
$\mathrm{C} 5-\mathrm{C} 6$ & $1.394(2)$ & $\mathrm{C} 23-\mathrm{C} 24$ & $1.393(2)$
\end{tabular}




\begin{tabular}{|c|c|c|c|}
\hline $\mathrm{C} 6-\mathrm{C} 7$ & $1.4462(18)$ & $\mathrm{C} 24-\mathrm{C} 25$ & $1.4437(19)$ \\
\hline $\mathrm{C} 7-\mathrm{C} 8$ & $1.3940(19)$ & $\mathrm{C} 25-\mathrm{C} 26$ & $1.3928(18)$ \\
\hline $\mathrm{C} 7-\mathrm{C} 12$ & $1.4113(19)$ & $\mathrm{C} 25-\mathrm{C} 30$ & $1.4142(19)$ \\
\hline $\mathrm{C} 8-\mathrm{H} 8$ & 0.9500 & $\mathrm{C} 26-\mathrm{H} 26$ & 0.9500 \\
\hline $\mathrm{C} 8-\mathrm{C} 9$ & $1.3890(19)$ & $\mathrm{C} 26-\mathrm{C} 27$ & 1.3909 (19) \\
\hline $\mathrm{C} 9-\mathrm{C} 10$ & $1.412(2)$ & $\mathrm{C} 27-\mathrm{C} 28$ & $1.4120(19)$ \\
\hline $\mathrm{C} 9-\mathrm{C} 13$ & $1.4890(19)$ & $\mathrm{C} 27-\mathrm{C} 31$ & $1.4875(18)$ \\
\hline $\mathrm{C} 10-\mathrm{C} 11$ & $1.3817(19)$ & $\mathrm{C} 28-\mathrm{C} 29$ & $1.3846(19)$ \\
\hline $\mathrm{C} 11-\mathrm{H} 11$ & 0.9500 & $\mathrm{C} 29-\mathrm{H} 29$ & 0.9500 \\
\hline $\mathrm{C} 11-\mathrm{C} 12$ & 1.3867 (19) & $\mathrm{C} 29-\mathrm{C} 30$ & $1.383(2)$ \\
\hline $\mathrm{C} 13-\mathrm{C} 14$ & $1.390(2)$ & $\mathrm{C} 31-\mathrm{C} 32$ & $1.389(2)$ \\
\hline $\mathrm{C} 13-\mathrm{C} 18$ & $1.3975(19)$ & C31-C36 & $1.394(2)$ \\
\hline C14-H14 & 0.9500 & $\mathrm{C} 32-\mathrm{H} 32$ & 0.9500 \\
\hline $\mathrm{C} 14-\mathrm{C} 15$ & $1.384(2)$ & $\mathrm{C} 32-\mathrm{C} 33$ & $1.389(2)$ \\
\hline $\mathrm{C} 15-\mathrm{H} 15$ & 0.9500 & $\mathrm{C} 33-\mathrm{H} 33$ & 0.9500 \\
\hline $\mathrm{C} 15-\mathrm{C} 16$ & $1.380(2)$ & C33-C34 & $1.378(3)$ \\
\hline C16-H16 & 0.9500 & $\mathrm{C} 34-\mathrm{H} 34$ & 0.9500 \\
\hline $\mathrm{C} 16-\mathrm{C} 17$ & $1.386(2)$ & $\mathrm{C} 34-\mathrm{C} 35$ & $1.379(3)$ \\
\hline $\mathrm{C} 17-\mathrm{H} 17$ & 0.9500 & $\mathrm{C} 35-\mathrm{H} 35$ & 0.9500 \\
\hline $\mathrm{C} 17-\mathrm{C} 18$ & $1.381(2)$ & $\mathrm{C} 35-\mathrm{C} 36$ & $1.379(2)$ \\
\hline $\mathrm{C} 18-\mathrm{H} 18$ & 0.9500 & $\mathrm{C} 36-\mathrm{H} 36$ & 0.9500 \\
\hline $\mathrm{C} 1-\mathrm{N} 1-\mathrm{H} 1$ & 125.5 & $\mathrm{C} 19-\mathrm{N} 3-\mathrm{H} 3 \mathrm{~A}$ & 125.5 \\
\hline $\mathrm{C} 12-\mathrm{N} 1-\mathrm{H} 1$ & 125.5 & $\mathrm{C} 30-\mathrm{N} 3-\mathrm{H} 3 \mathrm{~A}$ & 125.5 \\
\hline $\mathrm{C} 12-\mathrm{N} 1-\mathrm{C} 1$ & $109.05(11)$ & $\mathrm{C} 30-\mathrm{N} 3-\mathrm{C} 19$ & $109.05(11)$ \\
\hline $\mathrm{O} 1-\mathrm{N} 2-\mathrm{C} 10$ & $119.42(12)$ & $\mathrm{O} 3-\mathrm{N} 4-\mathrm{O} 4$ & $122.28(12)$ \\
\hline $\mathrm{O} 2-\mathrm{N} 2-\mathrm{O} 1$ & $122.57(13)$ & $\mathrm{O} 3-\mathrm{N} 4-\mathrm{C} 28$ & $119.53(12)$ \\
\hline $\mathrm{O} 2-\mathrm{N} 2-\mathrm{C} 10$ & $118.00(13)$ & $\mathrm{O} 4-\mathrm{N} 4-\mathrm{C} 28$ & $118.17(12)$ \\
\hline $\mathrm{N} 1-\mathrm{C} 1-\mathrm{C} 2$ & $129.30(13)$ & $\mathrm{N} 3-\mathrm{C} 19-\mathrm{C} 20$ & $129.19(13)$ \\
\hline $\mathrm{N} 1-\mathrm{C} 1-\mathrm{C} 6$ & $109.00(12)$ & $\mathrm{N} 3-\mathrm{C} 19-\mathrm{C} 24$ & $109.16(12)$ \\
\hline $\mathrm{C} 2-\mathrm{C} 1-\mathrm{C} 6$ & $121.70(13)$ & $\mathrm{C} 20-\mathrm{C} 19-\mathrm{C} 24$ & $121.64(14)$ \\
\hline $\mathrm{C} 1-\mathrm{C} 2-\mathrm{H} 2$ & 121.4 & $\mathrm{C} 19-\mathrm{C} 20-\mathrm{H} 20$ & 121.3 \\
\hline $\mathrm{C} 3-\mathrm{C} 2-\mathrm{C} 1$ & $117.14(14)$ & $\mathrm{C} 21-\mathrm{C} 20-\mathrm{C} 19$ & $117.45(14)$ \\
\hline $\mathrm{C} 3-\mathrm{C} 2-\mathrm{H} 2$ & 121.4 & $\mathrm{C} 21-\mathrm{C} 20-\mathrm{H} 20$ & 121.3 \\
\hline $\mathrm{C} 2-\mathrm{C} 3-\mathrm{H} 3$ & 119.0 & $\mathrm{C} 20-\mathrm{C} 21-\mathrm{H} 21$ & 119.1 \\
\hline $\mathrm{C} 2-\mathrm{C} 3-\mathrm{C} 4$ & $121.97(13)$ & $\mathrm{C} 20-\mathrm{C} 21-\mathrm{C} 22$ & $121.89(14)$ \\
\hline $\mathrm{C} 4-\mathrm{C} 3-\mathrm{H} 3$ & 119.0 & $\mathrm{C} 22-\mathrm{C} 21-\mathrm{H} 21$ & 119.1 \\
\hline $\mathrm{C} 3-\mathrm{C} 4-\mathrm{H} 4$ & 119.7 & $\mathrm{C} 21-\mathrm{C} 22-\mathrm{H} 22$ & 119.7 \\
\hline $\mathrm{C} 5-\mathrm{C} 4-\mathrm{C} 3$ & $120.65(14)$ & $\mathrm{C} 23-\mathrm{C} 22-\mathrm{C} 21$ & $120.61(15)$ \\
\hline $\mathrm{C} 5-\mathrm{C} 4-\mathrm{H} 4$ & 119.7 & $\mathrm{C} 23-\mathrm{C} 22-\mathrm{H} 22$ & 119.7 \\
\hline $\mathrm{C} 4-\mathrm{C} 5-\mathrm{H} 5$ & 120.6 & $\mathrm{C} 22-\mathrm{C} 23-\mathrm{H} 23$ & 120.6 \\
\hline $\mathrm{C} 4-\mathrm{C} 5-\mathrm{C} 6$ & $118.76(14)$ & $\mathrm{C} 22-\mathrm{C} 23-\mathrm{C} 24$ & $118.85(14)$ \\
\hline $\mathrm{C} 6-\mathrm{C} 5-\mathrm{H} 5$ & 120.6 & $\mathrm{C} 24-\mathrm{C} 23-\mathrm{H} 23$ & 120.6 \\
\hline $\mathrm{C} 1-\mathrm{C} 6-\mathrm{C} 7$ & $106.36(12)$ & $\mathrm{C} 19-\mathrm{C} 24-\mathrm{C} 25$ & $106.21(12)$ \\
\hline $\mathrm{C} 5-\mathrm{C} 6-\mathrm{C} 1$ & $119.77(13)$ & $\mathrm{C} 23-\mathrm{C} 24-\mathrm{C} 19$ & $119.53(13)$ \\
\hline $\mathrm{C} 5-\mathrm{C} 6-\mathrm{C} 7$ & $133.86(14)$ & $\mathrm{C} 23-\mathrm{C} 24-\mathrm{C} 25$ & $134.26(13)$ \\
\hline $\mathrm{C} 8-\mathrm{C} 7-\mathrm{C} 6$ & $133.64(13)$ & $\mathrm{C} 26-\mathrm{C} 25-\mathrm{C} 24$ & $133.90(13)$ \\
\hline $\mathrm{C} 8-\mathrm{C} 7-\mathrm{C} 12$ & $119.83(12)$ & $\mathrm{C} 26-\mathrm{C} 25-\mathrm{C} 30$ & $119.64(13)$ \\
\hline
\end{tabular}




\begin{tabular}{|c|c|c|c|}
\hline $\mathrm{C} 12-\mathrm{C} 7-\mathrm{C} 6$ & $106.53(12)$ & $\mathrm{C} 30-\mathrm{C} 25-\mathrm{C} 24$ & $106.46(12)$ \\
\hline $\mathrm{C} 7-\mathrm{C} 8-\mathrm{H} 8$ & 119.5 & $\mathrm{C} 25-\mathrm{C} 26-\mathrm{H} 26$ & 119.4 \\
\hline $\mathrm{C} 9-\mathrm{C} 8-\mathrm{C} 7$ & $120.95(13)$ & $\mathrm{C} 27-\mathrm{C} 26-\mathrm{C} 25$ & $121.17(12)$ \\
\hline $\mathrm{C} 9-\mathrm{C} 8-\mathrm{H} 8$ & 119.5 & $\mathrm{C} 27-\mathrm{C} 26-\mathrm{H} 26$ & 119.4 \\
\hline $\mathrm{C} 8-\mathrm{C} 9-\mathrm{C} 10$ & $116.54(12)$ & $\mathrm{C} 26-\mathrm{C} 27-\mathrm{C} 28$ & $116.44(12)$ \\
\hline $\mathrm{C} 8-\mathrm{C} 9-\mathrm{C} 13$ & $119.74(12)$ & $\mathrm{C} 26-\mathrm{C} 27-\mathrm{C} 31$ & $118.77(12)$ \\
\hline $\mathrm{C} 10-\mathrm{C} 9-\mathrm{C} 13$ & $123.46(12)$ & $\mathrm{C} 28-\mathrm{C} 27-\mathrm{C} 31$ & $124.67(12)$ \\
\hline $\mathrm{C} 9-\mathrm{C} 10-\mathrm{N} 2$ & $119.60(12)$ & $\mathrm{C} 27-\mathrm{C} 28-\mathrm{N} 4$ & $120.38(12)$ \\
\hline $\mathrm{C} 11-\mathrm{C} 10-\mathrm{N} 2$ & $115.52(12)$ & $\mathrm{C} 29-\mathrm{C} 28-\mathrm{N} 4$ & $114.84(12)$ \\
\hline $\mathrm{C} 11-\mathrm{C} 10-\mathrm{C} 9$ & $124.79(12)$ & $\mathrm{C} 29-\mathrm{C} 28-\mathrm{C} 27$ & $124.63(13)$ \\
\hline $\mathrm{C} 10-\mathrm{C} 11-\mathrm{H} 11$ & 121.7 & $\mathrm{C} 28-\mathrm{C} 29-\mathrm{H} 29$ & 121.6 \\
\hline $\mathrm{C} 10-\mathrm{C} 11-\mathrm{C} 12$ & $116.66(13)$ & $\mathrm{C} 30-\mathrm{C} 29-\mathrm{C} 28$ & $116.83(12)$ \\
\hline $\mathrm{C} 12-\mathrm{C} 11-\mathrm{H} 11$ & 121.7 & $\mathrm{C} 30-\mathrm{C} 29-\mathrm{H} 29$ & 121.6 \\
\hline $\mathrm{N} 1-\mathrm{C} 12-\mathrm{C} 7$ & $109.05(12)$ & $\mathrm{N} 3-\mathrm{C} 30-\mathrm{C} 25$ & $109.12(12)$ \\
\hline $\mathrm{N} 1-\mathrm{C} 12-\mathrm{C} 11$ & $129.75(13)$ & $\mathrm{N} 3-\mathrm{C} 30-\mathrm{C} 29$ & $129.64(12)$ \\
\hline $\mathrm{C} 11-\mathrm{C} 12-\mathrm{C} 7$ & $121.20(12)$ & $\mathrm{C} 29-\mathrm{C} 30-\mathrm{C} 25$ & $121.24(12)$ \\
\hline $\mathrm{C} 14-\mathrm{C} 13-\mathrm{C} 9$ & $121.11(12)$ & $\mathrm{C} 32-\mathrm{C} 31-\mathrm{C} 27$ & $119.84(13)$ \\
\hline $\mathrm{C} 14-\mathrm{C} 13-\mathrm{C} 18$ & $118.63(13)$ & $\mathrm{C} 32-\mathrm{C} 31-\mathrm{C} 36$ & $118.71(13)$ \\
\hline $\mathrm{C} 18-\mathrm{C} 13-\mathrm{C} 9$ & $120.21(12)$ & $\mathrm{C} 36-\mathrm{C} 31-\mathrm{C} 27$ & $121.33(13)$ \\
\hline $\mathrm{C} 13-\mathrm{C} 14-\mathrm{H} 14$ & 119.7 & $\mathrm{C} 31-\mathrm{C} 32-\mathrm{H} 32$ & 119.7 \\
\hline $\mathrm{C} 15-\mathrm{C} 14-\mathrm{C} 13$ & $120.60(14)$ & $\mathrm{C} 33-\mathrm{C} 32-\mathrm{C} 31$ & $120.58(15)$ \\
\hline $\mathrm{C} 15-\mathrm{C} 14-\mathrm{H} 14$ & 119.7 & $\mathrm{C} 33-\mathrm{C} 32-\mathrm{H} 32$ & 119.7 \\
\hline $\mathrm{C} 14-\mathrm{C} 15-\mathrm{H} 15$ & 119.8 & $\mathrm{C} 32-\mathrm{C} 33-\mathrm{H} 33$ & 120.0 \\
\hline $\mathrm{C} 16-\mathrm{C} 15-\mathrm{C} 14$ & $120.38(15)$ & $\mathrm{C} 34-\mathrm{C} 33-\mathrm{C} 32$ & $120.01(16)$ \\
\hline $\mathrm{C} 16-\mathrm{C} 15-\mathrm{H} 15$ & 119.8 & $\mathrm{C} 34-\mathrm{C} 33-\mathrm{H} 33$ & 120.0 \\
\hline $\mathrm{C} 15-\mathrm{C} 16-\mathrm{H} 16$ & 120.2 & $\mathrm{C} 33-\mathrm{C} 34-\mathrm{H} 34$ & 120.1 \\
\hline $\mathrm{C} 15-\mathrm{C} 16-\mathrm{C} 17$ & $119.61(14)$ & $\mathrm{C} 33-\mathrm{C} 34-\mathrm{C} 35$ & $119.77(15)$ \\
\hline $\mathrm{C} 17-\mathrm{C} 16-\mathrm{H} 16$ & 120.2 & $\mathrm{C} 35-\mathrm{C} 34-\mathrm{H} 34$ & 120.1 \\
\hline $\mathrm{C} 16-\mathrm{C} 17-\mathrm{H} 17$ & 119.9 & $\mathrm{C} 34-\mathrm{C} 35-\mathrm{H} 35$ & 119.7 \\
\hline $\mathrm{C} 18-\mathrm{C} 17-\mathrm{C} 16$ & $120.26(14)$ & $\mathrm{C} 34-\mathrm{C} 35-\mathrm{C} 36$ & $120.60(16)$ \\
\hline $\mathrm{C} 18-\mathrm{C} 17-\mathrm{H} 17$ & 119.9 & $\mathrm{C} 36-\mathrm{C} 35-\mathrm{H} 35$ & 119.7 \\
\hline $\mathrm{C} 13-\mathrm{C} 18-\mathrm{H} 18$ & 119.7 & $\mathrm{C} 31-\mathrm{C} 36-\mathrm{H} 36$ & 119.8 \\
\hline $\mathrm{C} 17-\mathrm{C} 18-\mathrm{C} 13$ & $120.52(14)$ & $\mathrm{C} 35-\mathrm{C} 36-\mathrm{C} 31$ & $120.31(15)$ \\
\hline $\mathrm{C} 17-\mathrm{C} 18-\mathrm{H} 18$ & 119.7 & $\mathrm{C} 35-\mathrm{C} 36-\mathrm{H} 36$ & 119.8 \\
\hline $\mathrm{O} 1-\mathrm{N} 2-\mathrm{C} 10-\mathrm{C} 9$ & $33.96(18)$ & $\mathrm{O} 3-\mathrm{N} 4-\mathrm{C} 28-\mathrm{C} 27$ & $35.93(18)$ \\
\hline $\mathrm{O} 1-\mathrm{N} 2-\mathrm{C} 10-\mathrm{C} 11$ & $-142.78(13)$ & $\mathrm{O} 3-\mathrm{N} 4-\mathrm{C} 28-\mathrm{C} 29$ & $-139.86(13)$ \\
\hline $\mathrm{O} 2-\mathrm{N} 2-\mathrm{C} 10-\mathrm{C} 9$ & $-147.07(14)$ & $\mathrm{O} 4-\mathrm{N} 4-\mathrm{C} 28-\mathrm{C} 27$ & $-145.47(13)$ \\
\hline $\mathrm{O} 2-\mathrm{N} 2-\mathrm{C} 10-\mathrm{C} 11$ & $36.19(18)$ & $\mathrm{O} 4-\mathrm{N} 4-\mathrm{C} 28-\mathrm{C} 29$ & $38.74(17)$ \\
\hline $\mathrm{N} 1-\mathrm{C} 1-\mathrm{C} 2-\mathrm{C} 3$ & $-178.83(13)$ & $\mathrm{N} 3-\mathrm{C} 19-\mathrm{C} 20-\mathrm{C} 21$ & $-177.36(13)$ \\
\hline $\mathrm{N} 1-\mathrm{C} 1-\mathrm{C} 6-\mathrm{C} 5$ & $179.82(11)$ & $\mathrm{N} 3-\mathrm{C} 19-\mathrm{C} 24-\mathrm{C} 23$ & $178.82(12)$ \\
\hline $\mathrm{N} 1-\mathrm{C} 1-\mathrm{C} 6-\mathrm{C} 7$ & $-0.43(14)$ & $\mathrm{N} 3-\mathrm{C} 19-\mathrm{C} 24-\mathrm{C} 25$ & $-0.84(15)$ \\
\hline $\mathrm{N} 2-\mathrm{C} 10-\mathrm{C} 11-\mathrm{C} 12$ & $176.07(11)$ & $\mathrm{N} 4-\mathrm{C} 28-\mathrm{C} 29-\mathrm{C} 30$ & $173.21(11)$ \\
\hline $\mathrm{C} 1-\mathrm{N} 1-\mathrm{C} 12-\mathrm{C} 7$ & $0.29(14)$ & $\mathrm{C} 19-\mathrm{N} 3-\mathrm{C} 30-\mathrm{C} 25$ & $-0.34(15)$ \\
\hline $\mathrm{C} 1-\mathrm{N} 1-\mathrm{C} 12-\mathrm{C} 11$ & $-179.86(13)$ & $\mathrm{C} 19-\mathrm{N} 3-\mathrm{C} 30-\mathrm{C} 29$ & $178.60(13)$ \\
\hline $\mathrm{C} 1-\mathrm{C} 2-\mathrm{C} 3-\mathrm{C} 4$ & $-0.8(2)$ & $\mathrm{C} 19-\mathrm{C} 20-\mathrm{C} 21-\mathrm{C} 22$ & $-1.6(2)$ \\
\hline $\mathrm{C} 1-\mathrm{C} 6-\mathrm{C} 7-\mathrm{C} 8$ & $-179.68(13)$ & $\mathrm{C} 19-\mathrm{C} 24-\mathrm{C} 25-\mathrm{C} 26$ & $-178.57(14)$ \\
\hline $\mathrm{C} 1-\mathrm{C} 6-\mathrm{C} 7-\mathrm{C} 12$ & $0.60(14)$ & $\mathrm{C} 19-\mathrm{C} 24-\mathrm{C} 25-\mathrm{C} 30$ & $0.62(14)$ \\
\hline
\end{tabular}




\begin{tabular}{|c|c|c|c|}
\hline $\mathrm{C} 2-\mathrm{C} 1-\mathrm{C} 6-\mathrm{C} 5$ & $0.67(19)$ & $\mathrm{C} 20-\mathrm{C} 19-\mathrm{C} 24-\mathrm{C} 23$ & $-0.4(2)$ \\
\hline $\mathrm{C} 2-\mathrm{C} 1-\mathrm{C} 6-\mathrm{C} 7$ & $-179.58(12)$ & $\mathrm{C} 20-\mathrm{C} 19-\mathrm{C} 24-\mathrm{C} 25$ & $179.97(12)$ \\
\hline $\mathrm{C} 2-\mathrm{C} 3-\mathrm{C} 4-\mathrm{C} 5$ & $0.7(2)$ & $\mathrm{C} 20-\mathrm{C} 21-\mathrm{C} 22-\mathrm{C} 23$ & $0.3(2)$ \\
\hline $\mathrm{C} 3-\mathrm{C} 4-\mathrm{C} 5-\mathrm{C} 6$ & $0.1(2)$ & $\mathrm{C} 21-\mathrm{C} 22-\mathrm{C} 23-\mathrm{C} 24$ & $1.0(2)$ \\
\hline $\mathrm{C} 4-\mathrm{C} 5-\mathrm{C} 6-\mathrm{C} 1$ & $-0.78(19)$ & $\mathrm{C} 22-\mathrm{C} 23-\mathrm{C} 24-\mathrm{C} 19$ & $-1.0(2)$ \\
\hline $\mathrm{C} 4-\mathrm{C} 5-\mathrm{C} 6-\mathrm{C} 7$ & $179.56(14)$ & $\mathrm{C} 22-\mathrm{C} 23-\mathrm{C} 24-\mathrm{C} 25$ & $178.56(14)$ \\
\hline $\mathrm{C} 5-\mathrm{C} 6-\mathrm{C} 7-\mathrm{C} 8$ & $0.0(3)$ & $\mathrm{C} 23-\mathrm{C} 24-\mathrm{C} 25-\mathrm{C} 26$ & $1.8(3)$ \\
\hline $\mathrm{C} 5-\mathrm{C} 6-\mathrm{C} 7-\mathrm{C} 12$ & $-179.70(14)$ & $\mathrm{C} 23-\mathrm{C} 24-\mathrm{C} 25-\mathrm{C} 30$ & $-178.97(15)$ \\
\hline $\mathrm{C} 6-\mathrm{C} 1-\mathrm{C} 2-\mathrm{C} 3$ & $0.13(19)$ & $\mathrm{C} 24-\mathrm{C} 19-\mathrm{C} 20-\mathrm{C} 21$ & $1.7(2)$ \\
\hline $\mathrm{C} 6-\mathrm{C} 7-\mathrm{C} 8-\mathrm{C} 9$ & $-178.43(13)$ & $\mathrm{C} 24-\mathrm{C} 25-\mathrm{C} 26-\mathrm{C} 27$ & $177.60(13)$ \\
\hline $\mathrm{C} 6-\mathrm{C} 7-\mathrm{C} 12-\mathrm{N} 1$ & $-0.56(14)$ & $\mathrm{C} 24-\mathrm{C} 25-\mathrm{C} 30-\mathrm{N} 3$ & $-0.18(14)$ \\
\hline $\mathrm{C} 6-\mathrm{C} 7-\mathrm{C} 12-\mathrm{C} 11$ & $179.58(11)$ & $\mathrm{C} 24-\mathrm{C} 25-\mathrm{C} 30-\mathrm{C} 29$ & $-179.23(12)$ \\
\hline $\mathrm{C} 7-\mathrm{C} 8-\mathrm{C} 9-\mathrm{C} 10$ & $-1.82(18)$ & $\mathrm{C} 25-\mathrm{C} 26-\mathrm{C} 27-\mathrm{C} 28$ & $0.96(19)$ \\
\hline $\mathrm{C} 7-\mathrm{C} 8-\mathrm{C} 9-\mathrm{C} 13$ & $172.60(12)$ & $\mathrm{C} 25-\mathrm{C} 26-\mathrm{C} 27-\mathrm{C} 31$ & $177.21(12)$ \\
\hline $\mathrm{C} 8-\mathrm{C} 7-\mathrm{C} 12-\mathrm{N} 1$ & $179.68(11)$ & $\mathrm{C} 26-\mathrm{C} 25-\mathrm{C} 30-\mathrm{N} 3$ & $179.14(11)$ \\
\hline $\mathrm{C} 8-\mathrm{C} 7-\mathrm{C} 12-\mathrm{C} 11$ & $-0.18(19)$ & $\mathrm{C} 26-\mathrm{C} 25-\mathrm{C} 30-\mathrm{C} 29$ & $0.09(19)$ \\
\hline $\mathrm{C} 8-\mathrm{C} 9-\mathrm{C} 10-\mathrm{N} 2$ & $-174.94(11)$ & $\mathrm{C} 26-\mathrm{C} 27-\mathrm{C} 28-\mathrm{N} 4$ & $-174.31(12)$ \\
\hline $\mathrm{C} 8-\mathrm{C} 9-\mathrm{C} 10-\mathrm{C} 11$ & $1.5(2)$ & $\mathrm{C} 26-\mathrm{C} 27-\mathrm{C} 28-\mathrm{C} 29$ & $1.0(2)$ \\
\hline $\mathrm{C} 8-\mathrm{C} 9-\mathrm{C} 13-\mathrm{C} 14$ & $55.80(18)$ & $\mathrm{C} 26-\mathrm{C} 27-\mathrm{C} 31-\mathrm{C} 32$ & $44.14(18)$ \\
\hline $\mathrm{C} 8-\mathrm{C} 9-\mathrm{C} 13-\mathrm{C} 18$ & $-121.42(14)$ & $\mathrm{C} 26-\mathrm{C} 27-\mathrm{C} 31-\mathrm{C} 36$ & $-131.90(14)$ \\
\hline $\mathrm{C} 9-\mathrm{C} 10-\mathrm{C} 11-\mathrm{C} 12$ & $-0.5(2)$ & $\mathrm{C} 27-\mathrm{C} 28-\mathrm{C} 29-\mathrm{C} 30$ & $-2.4(2)$ \\
\hline $\mathrm{C} 9-\mathrm{C} 13-\mathrm{C} 14-\mathrm{C} 15$ & $-177.70(13)$ & $\mathrm{C} 27-\mathrm{C} 31-\mathrm{C} 32-\mathrm{C} 33$ & $-175.23(13)$ \\
\hline $\mathrm{C} 9-\mathrm{C} 13-\mathrm{C} 18-\mathrm{C} 17$ & $177.90(13)$ & $\mathrm{C} 27-\mathrm{C} 31-\mathrm{C} 36-\mathrm{C} 35$ & $176.57(13)$ \\
\hline $\mathrm{C} 10-\mathrm{C} 9-\mathrm{C} 13-\mathrm{C} 14$ & $-130.18(14)$ & $\mathrm{C} 28-\mathrm{C} 27-\mathrm{C} 31-\mathrm{C} 32$ & $-139.94(14)$ \\
\hline $\mathrm{C} 10-\mathrm{C} 9-\mathrm{C} 13-\mathrm{C} 18$ & $52.60(18)$ & $\mathrm{C} 28-\mathrm{C} 27-\mathrm{C} 31-\mathrm{C} 36$ & $44.02(19)$ \\
\hline $\mathrm{C} 10-\mathrm{C} 11-\mathrm{C} 12-\mathrm{N} 1$ & $179.96(12)$ & $\mathrm{C} 28-\mathrm{C} 29-\mathrm{C} 30-\mathrm{N} 3$ & $-177.09(13)$ \\
\hline $\mathrm{C} 10-\mathrm{C} 11-\mathrm{C} 12-\mathrm{C} 7$ & $-0.20(18)$ & $\mathrm{C} 28-\mathrm{C} 29-\mathrm{C} 30-\mathrm{C} 25$ & $1.75(19)$ \\
\hline $\mathrm{C} 12-\mathrm{N} 1-\mathrm{C} 1-\mathrm{C} 2$ & $179.16(13)$ & $\mathrm{C} 30-\mathrm{N} 3-\mathrm{C} 19-\mathrm{C} 20$ & $179.86(13)$ \\
\hline $\mathrm{C} 12-\mathrm{N} 1-\mathrm{C} 1-\mathrm{C} 6$ & $0.10(14)$ & $\mathrm{C} 30-\mathrm{N} 3-\mathrm{C} 19-\mathrm{C} 24$ & $0.75(15)$ \\
\hline $\mathrm{C} 12-\mathrm{C} 7-\mathrm{C} 8-\mathrm{C} 9$ & $1.25(19)$ & $\mathrm{C} 30-\mathrm{C} 25-\mathrm{C} 26-\mathrm{C} 27$ & $-1.50(19)$ \\
\hline $\mathrm{C} 13-\mathrm{C} 9-\mathrm{C} 10-\mathrm{N} 2$ & $10.87(19)$ & $\mathrm{C} 31-\mathrm{C} 27-\mathrm{C} 28-\mathrm{N} 4$ & $9.68(19)$ \\
\hline $\mathrm{C} 13-\mathrm{C} 9-\mathrm{C} 10-\mathrm{C} 11$ & $-172.72(12)$ & $\mathrm{C} 31-\mathrm{C} 27-\mathrm{C} 28-\mathrm{C} 29$ & $-174.96(12)$ \\
\hline $\mathrm{C} 13-\mathrm{C} 14-\mathrm{C} 15-\mathrm{C} 16$ & $0.3(2)$ & $\mathrm{C} 31-\mathrm{C} 32-\mathrm{C} 33-\mathrm{C} 34$ & $-1.1(2)$ \\
\hline $\mathrm{C} 14-\mathrm{C} 13-\mathrm{C} 18-\mathrm{C} 17$ & $0.6(2)$ & $\mathrm{C} 32-\mathrm{C} 31-\mathrm{C} 36-\mathrm{C} 35$ & $0.5(2)$ \\
\hline $\mathrm{C} 14-\mathrm{C} 15-\mathrm{C} 16-\mathrm{C} 17$ & $-0.3(2)$ & $\mathrm{C} 32-\mathrm{C} 33-\mathrm{C} 34-\mathrm{C} 35$ & $-0.1(2)$ \\
\hline $\mathrm{C} 15-\mathrm{C} 16-\mathrm{C} 17-\mathrm{C} 18$ & $0.4(2)$ & $\mathrm{C} 33-\mathrm{C} 34-\mathrm{C} 35-\mathrm{C} 36$ & $1.5(2)$ \\
\hline $\mathrm{C} 16-\mathrm{C} 17-\mathrm{C} 18-\mathrm{C} 13$ & $-0.6(2)$ & $\mathrm{C} 34-\mathrm{C} 35-\mathrm{C} 36-\mathrm{C} 31$ & $-1.7(2)$ \\
\hline $\mathrm{C} 18-\mathrm{C} 13-\mathrm{C} 14-\mathrm{C} 15$ & $-0.4(2)$ & $\mathrm{C} 36-\mathrm{C} 31-\mathrm{C} 32-\mathrm{C} 33$ & $0.9(2)$ \\
\hline
\end{tabular}

Hydrogen-bond geometry $\left(A,{ }^{\circ}\right)$

\begin{tabular}{lllll}
\hline$D-\mathrm{H} \cdots A$ & $D-\mathrm{H}$ & $\mathrm{H} \cdots A$ & $D \cdots A$ & $D-\mathrm{H} \cdots A$ \\
\hline $\mathrm{N} 1-\mathrm{H} 1 \cdots \mathrm{O} 3^{\mathrm{i}}$ & $0.88(1)$ & $2.33(1)$ & $3.1825(16)$ & $162(1)$ \\
$\mathrm{N} 1-\mathrm{H} 1 \cdots \mathrm{O} 4^{\mathrm{i}}$ & $0.88(1)$ & $2.38(1)$ & $3.1331(17)$ & $143(1)$ \\
$\mathrm{N} 1-\mathrm{H} 1 \cdots \mathrm{N} 4^{\mathrm{i}}$ & $0.88(1)$ & $2.59(1)$ & $3.4610(17)$ & $168(1)$ \\
$\mathrm{N} 3-\mathrm{H} 3 A \cdots \mathrm{O} 1^{\mathrm{ii}}$ & $0.88(1)$ & $2.26(1)$ & $3.1079(18)$ & $162(1)$
\end{tabular}


$\mathrm{N} 3-\mathrm{H} 3 A \cdots \mathrm{O} 2^{\mathrm{ii}}$

$\mathrm{N} 3-\mathrm{H} 3 A \cdots \mathrm{N} 2^{\mathrm{ii}}$

Symmetry codes: (i) $-x+1,-y,-z+1$; (ii) $-x+2,-y+1,-z+1$.
$0.88(1)$

$0.88(1)$
$2.45(1)$

2.60 (1)
$3.2039(19)$

$3.4700(19)$
$143(1)$

$170(1)$

\section{.}

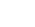

